\title{
Quelle position adopter face au patrimoine qui
} vient?

La médiation paysagère pour mettre en débat les temporalités

What position should be taken up with regard to upcoming heritage? The use of landscape mediation to discuss environmental temporalities

\section{Hervé Davodeau et Régis Barraud}

\section{(2) OpenEdition}

\section{Journals}

\section{Édition électronique}

URL : http://journals.openedition.org/developpementdurable/12277

DOI : 10.4000/developpementdurable. 12277

ISSN : 1772-9971

Éditeur

Association DD\&T

\section{Référence électronique}

Hervé Davodeau et Régis Barraud, «Quelle position adopter face au patrimoine qui vient? ", Développement durable et territoires [En ligne], Vol. 9, n² I Juin 2018, mis en ligne le 15 juin 2018, consulté le 02 mai 2019. URL : http://journals.openedition.org/developpementdurable/12277; DOI : 10.4000/developpementdurable.12277

Ce document a été généré automatiquement le 2 mai 2019.

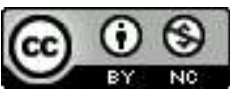

Développement Durable et Territoires est mis à disposition selon les termes de la licence Creative Commons Attribution - Pas d'Utilisation Commerciale 4.0 International. 


\title{
Quelle position adopter face au patrimoine qui vient?
}

\author{
La médiation paysagère pour mettre en débat les temporalités \\ What position should be taken up with regard to upcoming heritage? The use of \\ landscape mediation to discuss environmental temporalities
}

Hervé Davodeau et Régis Barraud

1 Cet article offre un retour synthétique et réflexif sur trois programmes de recherche ${ }^{1}$ sous l'angle des temporalités. Ayant pour point commun d'adopter le paysage comme entrée pour aborder les enjeux de patrimonialisation et de participation dans les démarches d'aménagement, ces programmes permettent de développer une réflexion sur les temporalités de l'aménagement. Pour éviter un propos trop général induit par la synthèse, nous avons choisi de placer au centre de l'analyse un des volets du programme PATRA : les épis de la Loire armoricaine à propos desquels nous questionnons la position $\mathrm{du}$ chercheur face au patrimoine qui vient, en construisant un scénario prospectif de patrimonialisation.

2 Plus fondamentalement, cet article est aussi pour nous l'occasion d'approfondir la question des « états de référence » en examinant comment ils sont à l'œuvre derrière les actions d'aménagement et de dés-aménagement analysées. Notre démarche centrée sur le paysage accorde une grande place à l'observation de terrain, mais ne se réduit pas à cette lecture : le paysage est aussi pour nous une façon d'aborder nos objets scientifiques, de les partager et les soumettre au débat public.

3 Après avoir replacé les épis dans leurs temporalités passées et analysé les usages et représentations dont ils sont le support aujourd'hui (1), nous élaborons une hypothèse patrimoniale à partir de nouveaux éléments du contexte territorial qui nous conduit à anticiper une possible controverse environnementale (2). Sur la base de cette anticipation, nous réfléchissons à la position que l'angle du paysage permet au chercheur d'adopter, en particulier pour mettre en débat les temporalités et les états de référence sous-jacents à l'action (3). 
Figure 1. Localisation et chronologie des aménagements de la Loire navigable

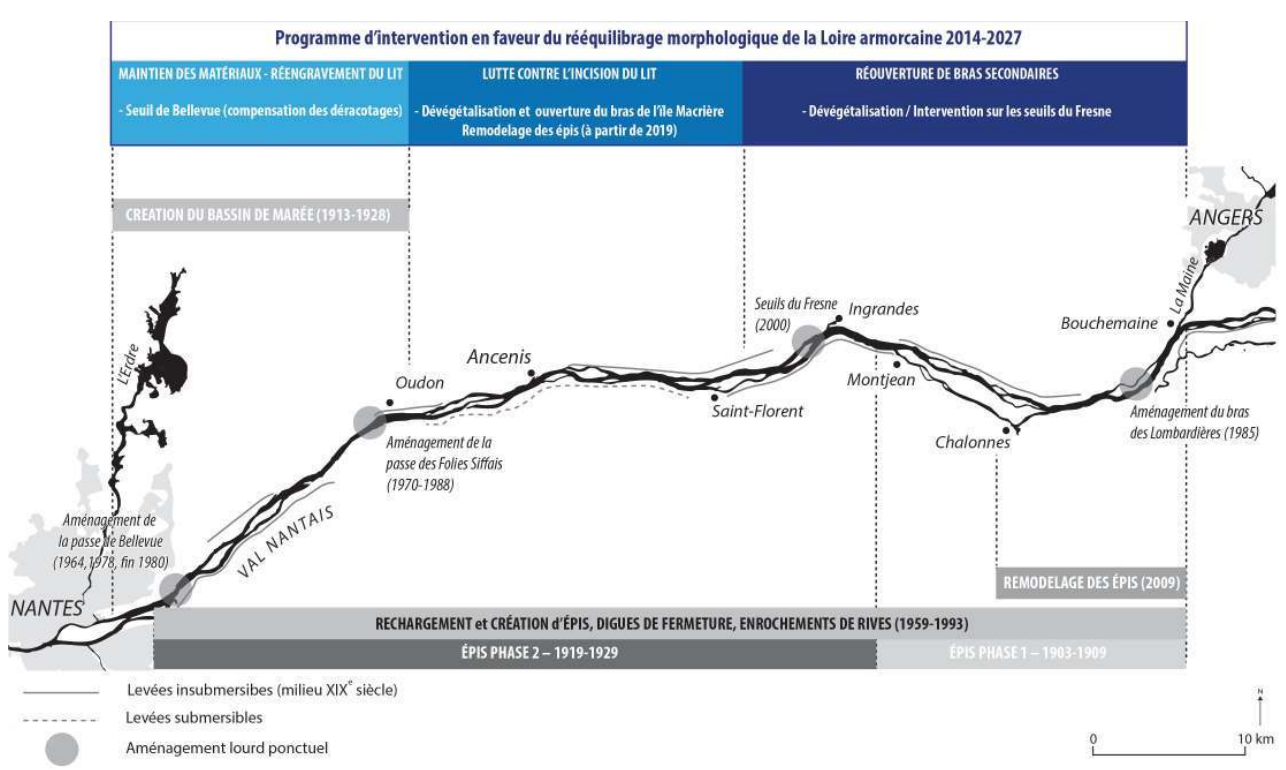

Réalisation : Barraud, 2016, Ruralités EA 2252.

Sources : Série S, fonds de l'ingénieur en chef (navigation Loire) - Archives départementales 14 VNF, 2016

\section{Le projet de la Loire navigable : trajectoire d'aménagement, références historiques et temporalités}

\subsection{Les épis de la Loire armoricaine : de l'aménagement initial au remodelage}

Les épis sont des ouvrages transversaux, positionnés « en peigne » dans le lit mineur du fleuve afin de le chenaliser et d'en assurer la navigabilité à l'étiage. Cette solution technique a été retenue au début du $\mathrm{xx}^{\mathrm{e}}$ siècle afin de surmonter la mobilité du chenal et la charge sédimentaire, vécues comme des contraintes majeures depuis la fin $d u \mathrm{XVIII}^{\mathrm{e}}$ siècle au moins. Au cours du XIX $x^{e}$ siècle, le débat fait rage entre les partisans de la canalisation et les «loiristes » privilégiant l'aménagement à "courant libre», plus modeste et fondé sur l'utilisation de la dynamique fluviale comme moteur de l'autoentretien du chenal. Ce débat technique et politique sur l'amélioration de la navigation dépasse largement les limites de la section armoricaine de la Loire, comme le démontrent les travaux de Le Marec (2000) et de Vauthier-Vézier (2007) pour la partie estuarienne, ou encore ceux de Lecœur (2011) pour la Loire moyenne. La mise en œuvre de cette chenalisation à courant libre s'effectue en deux phases. La première, expérimentale, donne lieu à l'aménagement d'une section de $14 \mathrm{~km}$ entre Bouchemaine et Montjean (1903-1908). La seconde (1922-1928) permet d'étendre l'installation des épis jusqu'aux portes de l'agglomération nantaise. La chenalisation de la Loire armoricaine prolonge les entreprises d'amélioration de la navigation de l'estuaire (canal maritime de la BasseLoire, 1892, bassin à marées, 1913). Une analyse détaillée de la mise en place des épis a déjà été publiée (Barraud et al., 2013). 
5 L'héritage sédimentaire, l'exploitation agricole de la bande active et la survenue de crues de printemps au moment même de la première phase d'établissement des épis contribuent à masquer le changement de fonctionnement hydro-sédimentaire du fleuve amorcé au cours de la deuxième partie du XIX ${ }^{\mathrm{e}}$ siècle (Charrier, 2000). Ainsi, au début du $\mathrm{xx}^{\mathrm{e}}$ siècle, les sociétés locales comme les ingénieurs perçoivent toujours la Loire comme un «fleuve de sable » caractérisé par des apports sédimentaires conséquents. En réalité, l'exhaussement du lit n'est alors plus d'actualité, mais les indicateurs de l'incision du lit ne seront validés par les experts qu'à partir du milieu du $x^{e}$ siècle : l'enfoncement spectaculaire du chenal et la déconnexion des annexes hydrauliques (bras secondaires, boires) ne seront réellement perçus qu'à partir des années soixante-dix, période au cours de laquelle l'exploitation intensive des granulats et les nouveaux travaux destinés à maintenir le chenal accentuent le phénomène. Les associations environnementales affrontent prioritairement la politique aménagiste à l'échelle du bassin de la Loire (construction de barrages) en médiatisant la défense de la Loire «dernier fleuve sauvage " (Pelosse et Micoud, 1993; Bonin, 2008; Rode, 2010). La mobilisation porte également sur la crise hydro-sédimentaire et la dégradation écologique de l'estuaire (bouchon vaseux, remontée du front salé, etc.). Les élus locaux relaient cette alerte en centrant leur propos sur les transformations paysagères liées à l'enfoncement du lit, très spectaculaire à l'étiage. Paradoxalement, on envisage d'abord des solutions techniques «modernes » pour pallier les effets de cette crise : l'aménagement de barrages mobiles est évoqué (sur le cours de la Loire ou aux confluences), tel celui de Pont-Rousseau à l'aval de la Sèvre nantaise.

Cette crise commencera à être prise en charge par les politiques publiques dans le cadre de la mise en œuvre du Plan Loire Grandeur nature (1994). L'enjeu du relèvement de la ligne d'eau d'étiage est clairement identifié dans cette première programmation, mais il faut attendre le deuxième Plan Loire pour assister à la mise en œuvre d'opérations spécifiques en Loire armoricaine. À nouveau, on procède à des expérimentations, comme le seuil noyé installé au Fresne-sur-Loire en 2003 dont l'efficacité hydraulique est jugée globalement satisfaisante, mais son aspect (boudin géotextile) et les courants générés suscitent toujours de nombreuses critiques. Le troisième Plan Loire (2007-2013) permet une nouvelle expérimentation là où les premiers épis ont été implantés au début du $\mathrm{xx}^{\mathrm{e}}$ siècle (entre Bouchemaine et Chalonnes) : près de 80 ouvrages sont modifiés sur une dizaine de kilomètres. Raccourcis de $25 \%$ de leur longueur (10 à 50 mètres) et abaissés de 0,5 mètre, les épis remodelés favorisent la remobilisation du sable stocké entre les épis. De manière complémentaire, l'abaissement ou le déplacement de digues longitudinales doit contribuer à la remise en eau des boires et des ports fluviaux. L'opération finement suivie durant plus de cinq ans semble porter ses fruits ${ }^{2}$, et le principe d'intervention sur les épis va être étendu à d'autres secteurs de la Loire armoricaine dans le cadre de la nouvelle programmation du Plan Loire (2015-2020). Ainsi, une intervention de remodelage des épis est prévue au niveau de la petite ville d'Ancenis, dans un contexte paysager plus urbanisé que celui de la première zone d'expérimentation. Désormais, l'intervention sur le lit mineur est qualifiée de « rééquilibrage morphologique » et doit se poursuivre jusqu'en 2027. Ce plan global s'articule avec un projet de restauration physique de l'estuaire. Les opérations prévues en Loire armoricaine traduisent l'affinement du diagnostic permis par les expérimentations successives et les suivis instrumentés. À l'aval d'Ancenis, il s'agira essentiellement d'assurer le maintien des sédiments dans le lit mineur et de compenser le déroctage du seuil de Bellevue (1976) ; le 
secteur d'Ancenis fera l'objet d'actions destinées à lutter contre l'incision (remodelage d'épis, ouverture du bras de l'île Neuve Macrière). Enfin, à l'amont, le réaménagement des seuils du Fresne et l'ouverture de quelques bras secondaires compléteront les travaux conduits sur les épis en 2009.

7 Cette synthèse géohistorique permet de comprendre que la mise en place des épis s'inscrit dans des temporalités complexes qui traduisent la dimension hybride de la Loire armoricaine (Chouquer, 2008; Jacob, 2009). En effet, les formes et les représentations paysagères associées au fleuve résultent d'interactions entre nature et société sur le temps long. Les sociétés ligériennes ont transformé, par des aménagements successifs, la physionomie du fleuve en forçant ou bien en réorientant certains processus hydromorphologiques (Carcaud et Burnouf, 2001 et 2003) afin de tirer meilleur parti de l'exploitation du lit mineur (navigation, pêcheries, moulins, etc.) ou du lit majeur (agriculture, développement d'infrastructures de transport).

\subsection{Les références temporelles sous-tendues par le projet « Loire navigable »}

8 Au sujet de la restauration écologique, d'importants débats ont eu lieu quant aux états de référence et à leur pertinence pour guider l'intervention (Baron-Yelles, 2000 ; Bravard, 2003 ; Lévêque et Van der Leeuw, 2006 ; Bouleau et Pont, 2014). D’une manière générale est battue en brèche l'utilisation de l'état de référence historique comme objectif de restauration dont la finalité serait de ramener à un état antérieur les milieux (Dufour et Piégay, 2009). Mais notre propos ne portera pas précisément sur les états de référence tels qu'appréhendés dans le champ de l'écologie de la restauration. Nous chercherons plutôt à identifier et caractériser les figures temporelles utilisées dans le cadre précis de l'installation initiale des épis dans le projet de la Loire navigable, puis une centaine d'années plus tard dans les projets de rééquilibrage du lit. L'analyse est ici inspirée par les travaux de J.-P. Haghe qui a proposé en 2010 une grille d'analyse des discours permettant de repérer les champs d'expérience, les horizons d'attente et les régimes d'historicité sous-jacents aux projets d'aménagement et de gestion des cours d'eau.

Les projets d'amélioration de la navigation sur la Loire se sont succédé jusqu'au début du $\mathrm{xx}^{\mathrm{e}}$ siècle. Il n'est pas possible de les examiner de manière exhaustive dans le cadre de cet article. Le recours à des aménagements fixes implantés dans le lit mineur s'est imposé au fil du temps, mais, à la fin du XIX ${ }^{e}$ siècle, les promoteurs de la Loire navigable font surtout référence aux travaux d'entretien réguliers assurés entre le $\mathrm{xv}^{\mathrm{e}}$ siècle et la fin de l'Ancien Régime par la "communauté des marchands fréquentant la Loire » (Mantellier, 1864 ; Vauthier-Vézier, 2007). C'est l'ajustement régulier du chenal par le déblaiement des atterrissements (chevalage), la lutte contre les aménagements illégaux entravant la navigation, le pilotage et le balisage qui était perçu comme la solution pour permettre une circulation difficile, mais intense sur le fleuve.

10 Le projet porté par la société de la Loire navigable à partir de 1896 fait explicitement référence à un âge d'or de la batellerie qu'il conviendrait alors de "ressusciter " (Zimmerman, 1905). Toutefois celui-ci n'est pas précisément borné historiquement, bien que le milieu du XIX $x^{e}$ siècle semble constituer le point de rupture marquant la fin d'une économie de la navigation florissante et l'entrée en crise. Les commentateurs du projet de chenalisation font appel à des références variées : le rôle « des flottilles de gabares » dans la délivrance d'Orléans par Jeanne d'Arc en 1429, ou encore la descente de la Loire 
d'Orléans à Nantes en 1680 par Mme de Sévigné (Ducamp, 1900). Le développement éphémère de la navigation à vapeur principalement dédiée au transport de voyageurs à partir de 1822, et essentiellement jusqu'aux années 1850-1860, constitue un autre temps de référence évoqué dans les discours des partisans du renouveau du trafic fluvial. L'imagerie des steamers et de leurs roues à aubes, très diffusée et artialisée, permet d'ancrer profondément ce mythe de l'âge d'or de la navigation. En conséquence, sa crise est le plus souvent située au milieu du XIX ${ }^{\mathrm{e}}$ siècle et résulterait de la conjonction du développement du transport ferroviaire et de la dégradation des conditions de navigation par la combinaison du manque d'entretien du chenal et la surabondance du sable (Gallouedec, 1897). La crise de la Loire fluviale est bien restituée par Maurice Schwob (1900), directeur du journal Le Phare et acteur clé de la «Loire navigable ». Ce dernier évoque un fleuve malade dont il faut hâter la guérison et "éviter les rechutes [en le soumettant] à une hygiène sévère ». La remédiation à la crise passe alors par une forme d'intervention chirurgicale des ingénieurs. Il faut donc saisir ici des temporalités complexes: celle de l'action de restauration des conditions de navigation, celle de la réponse du fleuve à la mise en place des épis (incertitude), et celle de la pérennisation du chenal à moyen/long terme par l'auto-curage attendu.

11 Par ailleurs, la mise en œuvre du projet de navigabilité met en jeu d'autres « espacestemps » qui témoignent d'un régime moderne d'historicité, dont l'horizon d'attente est caractérisé par le désir de progrès social et économique (Haghe, 2010). En effet, la Loire navigable est motivée par l'utopie du Grand Central (Gallouedec, op.cit.), c'est-à-dire le développement d'un axe de transport majeur reliant la façade atlantique à l'Europe centrale industrielle. Cet axe est censé favoriser le développement du port de Nantes dans un contexte commercial de plus en plus concurrentiel sur le plan international (ouverture du canal de Panama). La Loire navigable doit également contribuer au développement et à l'autonomie économique des régions ligériennes dont les productions sont parfois exportées via d'autres ports (Manche, Méditerranée). Enfin, cet axe de promotion du développement ligérien mobilise le souvenir de la défaite de 1870 et s'appuie sur une rhétorique patriotique (la Loire « fleuve national »).

12 Au final, la mise en place d'un chenal à courant libre au début $d u x^{e}$ siècle n'est pas réductible à un simple projet d'ingénieur, il s'agit d'un projet politique d'ampleur, inscrit dans des références temporelles variées. La chenalisation à l'aide d'épis se veut moderne et utilitariste, répondant à une crise et poursuivant un progrès. Pour autant, ce projet puise dans un répertoire d'images et de symboles issus d'un passé idéalisé. Par ailleurs, contrairement à ce qu'aurait engagé la mise en place d'un canal, la technique de l'ingénieur est combinée à une tentative de pilotage des processus hydro-sédimentaires pour assurer l'auto-entretien du chenal. Le durcissement de l'infrastructure par les rechargements d'épis et des berges en enrochements modifiera cette configuration et accélérera la crise hydro-sédimentaire perçue à partir des années 1970. Celle-ci traduira l'échec de cette tentative de contrôle d'un fleuve à fond mobile et l'essoufflement de l'utopie du développement économique fondé sur un axe de transport fluvial structurant.

\subsection{Les références temporelles contemporaines des projets de restauration écologique et paysagère}

13 Une centaine d'années après l'émergence du projet de la Loire navigable, le trop de sable a laissé place à une pénurie de sable. Paradoxalement, l'image du fleuve de sable est 
toujours forte, car l'enfoncement du chenal le rend encore plus visible. Mais ce fleuve de sable tend à se changer en "fleuve vert ", caractérisé par la végétalisation des annexes hydrauliques, des îles et des grèves, voire de certains ouvrages hydrauliques. Cette nouvelle crise produit de nouveaux discours et de nouvelles représentations au service d'un projet de restauration qui s'appuie également sur des références historiques pour fonder l'action publique actuelle de rééquilibrage du lit de la Loire. Le récit élaboré dans la plaquette "Si les épis m'étaient contés » (GIP Loire, 2009) articule trois temps. Le premier, situé au début du $\mathrm{xx}^{\mathrm{e}}$ siècle, est celui de la mise en place des épis, le deuxième est celui de la crise hydro-sédimentaire, dont le bornage temporel est imprécis, mais relié à des facteurs bien identifiés (chenalisation, extraction de sable), puis le troisième temps est le temps de la remédiation par l'expérimentation. Cette dernière phase intègre la temporalité de l'action publique (de l'enquête publique aux travaux et suivis) et l'articule de manière prudente avec celle de la réponse $d u$ fleuve. Les expérimentations contemporaines, initiées à partir de 2003 (seuils du Fresne) sont caractérisées par un accompagnement très pointu des travaux réalisés par des suivis instrumentés et des modélisations avancées, permettant des prédictions de la réponse du fleuve, mais aussi un réajustement permanent de l'intervention dans le lit mineur. Cette approche illustre bien le basculement du régime moderne d'historicité à celui du présentisme tel que le démontre d'une manière plus globale J.-P. Haghe (2010):»Il ne s'agit plus seulement de discipliner les eaux par des aménagements, mais d'accompagner les variations des caractéristiques normées des eaux pour pouvoir les réguler en permanence, dans l'immédiateté. "

Au-delà de sa dimension écologique, cette crise perturbe les usages locaux et modifie les paysages. Elle donne donc lieu à une mise en récit par les riverains avec ses propres temporalités. Ainsi, le discours de l'association "Comité Loire de Demain» (CLD) positionne souvent le démarrage de la crise au milieu des années soixante-dix, l'année 1976 étant régulièrement évoquée comme point de bascule. C'est à cette époque que des travaux lourds d'entretien du chenal (déroctage de seuils rocheux, suppression d'ouvrages médiévaux modifiant la répartition des flux entre les bras) auraient renforcé le processus d'enfoncement du lit dans un contexte d'extraction sablière intense. Le récit de l'association intègre également une critique de l'inertie des politiques publiques. La rhétorique de la Loire armoricaine abandonnée est renforcée par l'inefficacité et la grande modestie des actions de restauration entreprise depuis le premier Plan Loire (1994) jusqu'au lancement de l'opération de remodelage des épis. Le diagnostic de l'enfoncement de la ligne d'eau est repris par l'association sur la base des données issues des mesures de niveau depuis 1900. En 2009, avant le remodelage, l'association estimait que l'inaction publique conduirait à un retour à l'équilibre d'ici 400 ans. À partir d'une approche critique de cette politique publique, mais aussi en fonction de connaissances scientifiques solides, le CLD développe des propositions d'interventions complémentaires, en particulier celle des « duits filtrants ».

15 Contrairement aux documents de communication produits par les acteurs publics, les plaquettes d'information publiées par le CLD intègrent des références temporelles d'avant la mise en place des épis. La première est celle d'une Loire sauvage dont le lit présentait 128 sinuosités entre Nantes et Bouchemaine (alternance de mouilles et de hauts fonds). Cette référence est jugée utile pour comprendre les modalités du transit sédimentaire. La deuxième est celle des ouvrages médiévaux légers appelés duits et structurés par des lignes de pieux fascinés (moulins, pêcheries, péages). Enfin la troisième est celle des lignes de pieux de bois aménagées sur les littoraux dès l'Ancien Régime afin de lutter 
contre l'érosion. Ces différentes références techniques et historiques, surtout les deux premières, réexploitent en les adaptant des techniques anciennes en même temps qu'elles ancrent les rapports au fleuve dans une logique d'observation et d'ajustements réguliers. La dimension prospective des propositions du CLD traduit les attentes sociales de "réparation » du sinistre causé au fleuve. Les interventions alternatives présentées par l'association doivent entraîner des résultats significatifs dans les 20 années à venir. Enfin, dans une certaine mesure, le CLD reprend d'une manière consciente ou non une logique de contre-expertise et de mobilisation des savoirs locaux des riverains: la visite d'observation en bateau à partir du port de la Possonnière organisée en septembre 2016 par le CLD pour évaluer les résultats de l'opération de remodelage fait écho à celle demandée par les riverains dans les années 1920, suite à la deuxième étape d'installation des épis.

Compte tenu des moyens nouveaux et importants inscrits au Plan Loire 2015-2020 pour le rééquilibrage morphologique de la Loire armoricaine et estuarienne, l'approche très critique du CLD tend à s'adoucir désormais. Certaines de leurs propositions sont intégrées par les ingénieurs (ouverture de bras, intervention sur le seuil de Bellevue). Très progressivement, la concertation initiée depuis 2008 accorde plus de crédit aux connaissances des riverains, faisant du rééquilibrage de la Loire un objet de plus en plus hybride, entre modèles d'experts et savoirs locaux (expertise d'usage). L'opération de restauration prend alors la forme d'un projet politique de pilotage de la biodiversité au service, comme le suggère Blandin (2009), d'une nature désirée.

\subsection{Usages et perceptions}

Les épis sont aujourd'hui le support de pratiques sociales intenses sur ou autour de l'objet : pêche et amarrage des barques en aval à l'abri du courant, baignade en aval dans les bassines creusées par le fleuve, observation en immersion au milieu du lit (en pointe des épis), récoltes de bois morts piégés dans l'épi pour préparer les feux (barbecue), etc. Ces usages détournés ne sont pas en lien avec la fonction initiale des ouvrages (concentrer le courant pour creuser le chenal), ce qui contribue à la méconnaissance des riverains de cette histoire récente de la navigation à gros gabarits.

Les enquêtes conduites auprès des riverains démontrent cette méconnaissance (les épis sont peu nommés par exemple) et donnent à entendre plusieurs réinterprétations fonctionnelles : pêcheries, jetées, barrages pour rehausser la ligne d'eau ou atténuer les inondations (Barraud et al., 2013-1). Surtout, ces enquêtes témoignent de la domination de l'image du « dernier fleuve sauvage d'Europe » à laquelle les épis sont intégrés malgré la contradiction a priori évidente. L'écrasante majorité des personnes enquêtées sont d'accord avec l'expression "Loire sauvage", y compris celles qui choisissent une photographie à épis comme la plus représentative des paysages ligériens. Ce résultat témoigne de l'imbrication de trois regards : les épis sont tolérés dans un "paysage-vu » tiraillé entre le "paysage-vécu » où ils sont acceptés et le " paysage-su » duquel ils sont rejetés (Davodeau et al., 2013). Ce cadre du paysage-su dans lequel ces objets n'ont pas leur place est construit par les connaissances expertes qui font converger un modèle scientifique et un modèle esthétique autour de l'image du fleuve sauvage à propos duquel nous reviendrons. Pour reprendre la typologie de R. Larrère (1997), nos enquêtes (Barraud et al., 2012, 2013) démontrent à la fois le poids du regard «informé » sur les effets néfastes que ces aménagements provoquent sur les milieux (l'assèchement des bras, 
corollaire de l'enfoncement) et celui du regard «formé » par le modèle esthétique du fleuve sauvage. Cette représentation sous-tend aujourd'hui les politiques de gestion du fleuve, dont le paradigme est réorienté depuis le milieu des années quatre-vingt-dix autour de la restauration écologique (plan Loire Grandeur Nature à partir de 1994) et qui est conforté à travers le Plan Loire IV (2014-20).

\section{L'hypothèse patrimoniale}

\subsection{Nouveaux éléments de contexte}

19 L'exercice de prospective prend en considération plusieurs éléments nouveaux qui font évoluer le contexte territorial dans lequel les épis prennent place: la navigation touristique de gros gabarit qui profite du chenal à épis et qui a été relancée en Loire armoricaine en $2015^{3}$, le marketing territorial exercé par les offices de tourisme autour d'une "Autre Loire ${ }^{4}$ " où les épis figurent en bonne place pour faire valoir l'image spécifique de la Loire armoricaine (Carcaud, Davodeau, 2013), la création des communes nouvelles qui les conduit à mobiliser la ressource paysagère dans la construction de leur identité territoriale 5 . Dans ce contexte, nous observons les signes d'une reconnaissance des épis (figure 2) qui, à défaut d'alimenter un réel processus de patrimonialisation, construit un premier travail de mémoire : des signalétiques leur sont dédiées sur le port restauré de La Possonnière, dans l'île touristique de Béhuard, et le long de la promenade Julien-Gracq à Saint-Florent-le-Vieil ${ }^{6}$.

Dans ce contexte, qui semble redonner aux épis une plus grande visibilité, prend place le programme de rééquilibrage du lit de la Loire entre les Ponts-de-Cé et Nantes (2014-2027) qui prolonge en aval la première expérimentation de remodelage des épis et l'accentue même en envisageant la suppression de certains ouvrages ${ }^{7}$. Ces deux éléments de contexte nous conduisent à entrevoir une éventuelle controverse à venir autour des épis. 
Figure 2. Indice d'esthétisation des épis à l'entrée de Bouchemain

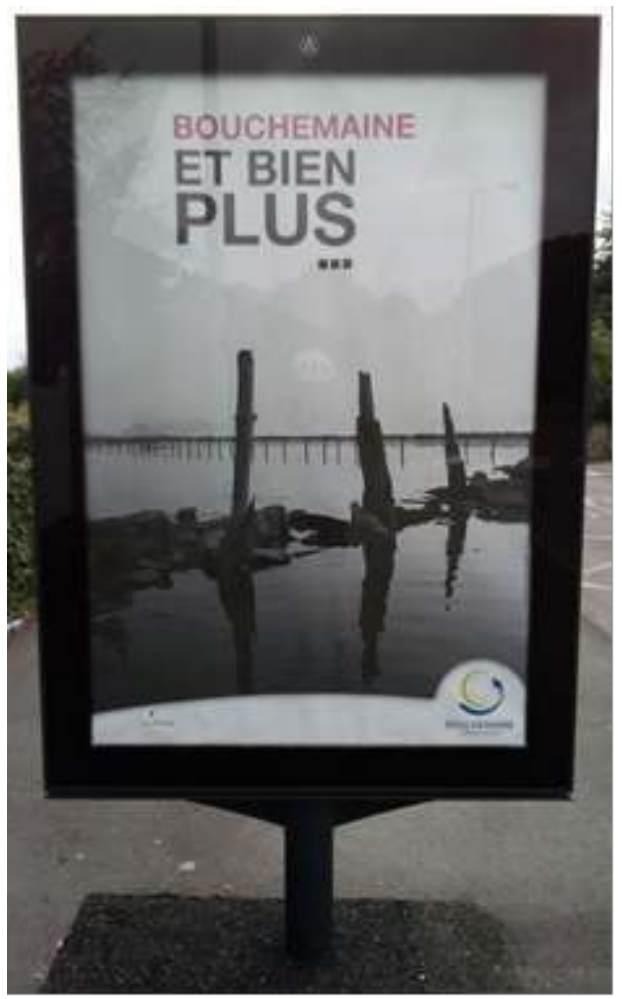

Source : Davodeau, avril 2017

\subsection{Anticiper une controverse environnementale}

21 Cette controverse pourrait résulter de la mise en tension de deux visions du fleuve : le mythe de l'ensauvagement, et celui de la navigation. À défaut de constituer des scénarios de gestion (même fictifs), ils agissent comme deux systèmes de valeurs sur la politique de restauration écologique qui s'impose aujourd'hui, et dont l'orientation à venir reflétera la capacité relative de ces deux paradigmes à infléchir la gestion du fleuve.

Celui de l'ensauvagement repose sur l'image du «fleuve sauvage » et porte le projet de sa « libération », en accord avec une radicalisation de la représentation de la nature, propre à l'idée du wilderness (Barraud, 2011). Cette Loire s'oppose au modèle culturaliste du fleuve royal (celui des châteaux) et pourra se traduire par une accentuation de la politique actuelle de restauration écologique, voire justifier une déclassification de la section navigable entre Angers et Nantes. Cette projection est envisageable dans un contexte économique de crise favorable à une politique de non-intervention (ensauvagement des épis par abandon de l'entretien du chenal), ou à l'inverse d'un contexte économique très favorable qui pourrait permettre la suppression intégrale des épis. Difficilement concevable à l'échelle globale du fleuve, ce scénario marque dès aujourd'hui certains lieux pouvant être l'objet d'un ensauvagement, mais de façon ponctuelle et expérimentale.

Celui de la navigation recouvre une réalité nouvelle depuis deux ans avec la circulation du bateau à aube Loire Princesse, dont le style cherche à rappeler ceux de la compagnie Les messageries de la Loire, et dans le sillage duquel pourraient se développer d'autres 
projets, tant pour le tourisme, la plaisance, qu'éventuellement le fret ${ }^{8}$. Ce projet politique nécessiterait de conserver les épis et pourrait s'appuyer sur une valorisation mémorielle de la navigation et une patrimonialisation des ouvrages dédiés. Cette hypothèse prendrait sens dans un contexte de revendication d'une Loire armoricaine qui pourrait éventuellement être l'objet d'une institutionnalisation (projet de PNR ?). La navigation serait alors un élément parmi d'autres d'un territoire ligérien qui chercherait à se démarquer - par son caractère industrieux, urbain, populaire - vis-à-vis de la Loire saumuroise et tourangelle en amont. Ce scénario prolongerait en l'actualisant le mythe de la Loire navigable en misant sur une navigation à gros gabarits (citadine et élitiste), au détriment des embarcations légères de la navigation populaire (car les ouvrages qui facilitent la navigation des gros gabarits rendent plus difficile celle des petits).

Comment peser relativement ces deux influences sur la gestion à venir du fleuve? La lecture des documents de programmation des travaux nous conduit à discerner deux phases dans le processus de restauration écologique: une première marquée par une approche techniciste de l'ingénierie écologique, exercée à travers des expérimentations locales contestées (le seuil du Fresnes en 2004) et sous maîtrise d'ouvrage de l'État par l'intermédiaire de V.N.F (devant assumer une mission a priori contraire à ses prérogatives), et une seconde marquée par une approche plus intégrée de l'aménagement, plus attentive aux usages et paysages ${ }^{9}$. Cette seconde qui s'ouvre peutêtre reflète une nouvelle gouvernance moins descendante, partagée entre l'État et les acteurs locaux, marquée en particulier par l'apport de la contre-expertise exercée par l'association Loire de demain ${ }^{10}$ prenant la défense d'une Loire armoricaine «sinistrée et abandonnée ». Alors que dans la première phase de l'expérimentation le remodelage concernait tous les épis en intervenant de façon identique (abaissement et raccourcissement), la seconde différenciera les épis à remodeler, supprimer, conserver.

\section{La médiation paysagère pour mettre en débat les temporalités}

\subsection{Anticiper une recherche-action et se positionner par le paysage}

Ce travail de sélection s'appuiera sur une expertise technique, mais la volonté affichée de concertation avec les acteurs locaux permet aussi d'envisager une démarche bottom-up à partir des pratiques locales. Si tel est le cas, une commande pourrait être formulée par les gestionnaires auprès des chercheurs pour éclairer les choix d'aménagement au regard des usages et perceptions des lieux. Sur la base de cette expertise d'usage, quels ouvrages supprimer, remodeler, conserver? Quels aménagements promouvoir pour les préserver, voire les développer? Si les formes héritées sont celles des épis de navigation, peut-on en imaginer d'autres ou du moins les adapter aux nouveaux usages? Que seraient des épis de baignade, de pêche, d'observation, d'accostage ? Comment penser la multifonctionnalité de ces objets? Ces questions de conception nécessiteraient d'articuler la recherche-action à des ateliers pédagogiques dans lesquels les étudiants pourraient expérimenter une situation de médiation paysagère (Dubois, 2009 ${ }^{11}$; Davodeau, 2012).

Cette démarche traduit une posture particulière que le paysage nous permet de tenir aussi bien vis-à-vis de la commande (ici fictive) que de nos questions scientifiques. Nous considérons le paysage comme une entrée pour replacer nos objets dans leurs trajectoires historiques (Barraud et al., 2013-2), pour questionner les processus de patrimonialisation 
et de restauration écologique, notamment en réinterrogeant les états de référence sousjacents aux représentations et à l'action publique. Par ailleurs, l'analyse des paysages est aussi un outil pour saisir les pratiques sociales sur les lieux observés. Il est donc pour nous une façon de penser le patrimoine (par le paysage) en l'abordant de façon dynamique (les trajectoires paysagères) et par le vécu (le paysage ordinaire). Cette posture de recherche est distanciée, car elle propose un point de vue critique sur les processus de patrimonialisation et de restauration écologique (radicalisation des représentations de la nature, archétypes, élitisme, verrouillage de l'expertise), mais elle est aussi impliquée dans la mesure où - par l'angle d'une géographie sociale - elle permet de défendre les usages de proximité et de complexifier l'expertise (pas seulement naturaliste). Cette posture scientifique qui nous a guidés au cours des programmes PATRA (Davodeau et al., 2012) et REPPAVAL (Barraud et Germaine, 2017) ne nous conduit pas pour autant à prendre parti pour défendre la valeur patrimoniale des épis, mais plutôt à souligner la nature relationnelle propre aux « communs » paysagers (Sgard, 2010). Notre implication ne consiste donc pas à sauvegarder les épis en l'état, mais à assurer le maintien, voire le développement des usages sur ces lieux, selon une approche relationnelle du paysage, moins attentive à l'objet lui-même (dont l'enjeu serait la conservation formelle), qu'à l'intensité des relations socio-spatiales qui se nouent à travers lui ${ }^{12}$.

Dans cette même perspective consistant à penser le patrimoine "par le paysage ", la participation peut être conduite " par le paysage »; c'est la proposition de la médiation paysagère expérimentée dans le cadre du programme PDD2 à Villandry pour travailler avec les habitants sur le réaménagement du centre-bourg (Montembault et al., 2015). Avec recul, nous pouvons relire cette expérience à la lumière des temporalités, puisque la question du temps est directement posée dans le principal enjeu d'aménagement formulé par les habitants, autour duquel nous avons construit avec eux la commande (la revitalisation du centre-bourg "à l'ombre du château »). Derrière cet enjeu spatial (deux espaces qui se côtoient sans suffisamment de lien) se jouent deux temporalités : celle du bourg (quotidienne) et celle du château et ses jardins (événementielle). Ces deux temporalités sont problématiques dans la traversée du bourg, le stationnement, le déclin de l'activité commerciale. La relecture réflexive de notre démarche sous cet angle nous permet également de mettre en lumière les enjeux temporels de la mobilisation pour les ateliers, et de leur animation: quand les planifier, comment penser le déroulé de la démarche par phase et surtout les transitions entre les ateliers (pour les participants qui " prennent le train en route »)?

\subsection{Mettre en débat les temporalités}

La formalisation graphique (figure 3) de la trajectoire géohistorique des épis (Davodeau et al., 2013) permet d'offrir un support d'échange pour discuter des références temporelles sous-tendues par les actions d'aménagement (Bercovitz, 2015). Ces représentations matérielles permettent de contextualiser ces objets de l'ingénierie dans le cadre plus politique des projets globaux de valorisation de la vallée de la Loire armoricaine. Ainsi appréhendés dans leur trajectoire paysagère, les épis apparaissent comme le dernier grand acte d'aménagement de la Loire navigable, derniers témoins d'un projet très vite concurrencé par le transport ferroviaire. Leur présence dans le paysage tient à l'inertie propre aux trajectoires paysagères, dont les processus d'évolution peuvent être éteints, mais les effets longtemps visibles (et même davantage aujourd'hui qu'hier avec 
l'abaissement du lit auquel ils ont contribué). La faible valeur patrimoniale des épis (nous la pensons émergente) reflète un état de référence liée à la navigation : ces ouvrages sont associés aux bateaux à vapeur et pétroliers (une histoire mal connue des riverains) et non à la batellerie traditionnelle (celle des gabares et des toues) qui - elle - est l'objet d'une patrimonialisation. Ne trouvant pas encore leur place dans le récit patrimonial de la navigation plus contemporaine, les épis sont alors captés par celui, bien plus puissant, du « fleuve sauvage », comme le démontrent nos enquêtes (Barraud et al., 2012 et 2013-1).

Figure 3. Représentation imagée du passé (en amont, à gauche) au présent (en aval, à droite) des paysages de Loire armoricaine

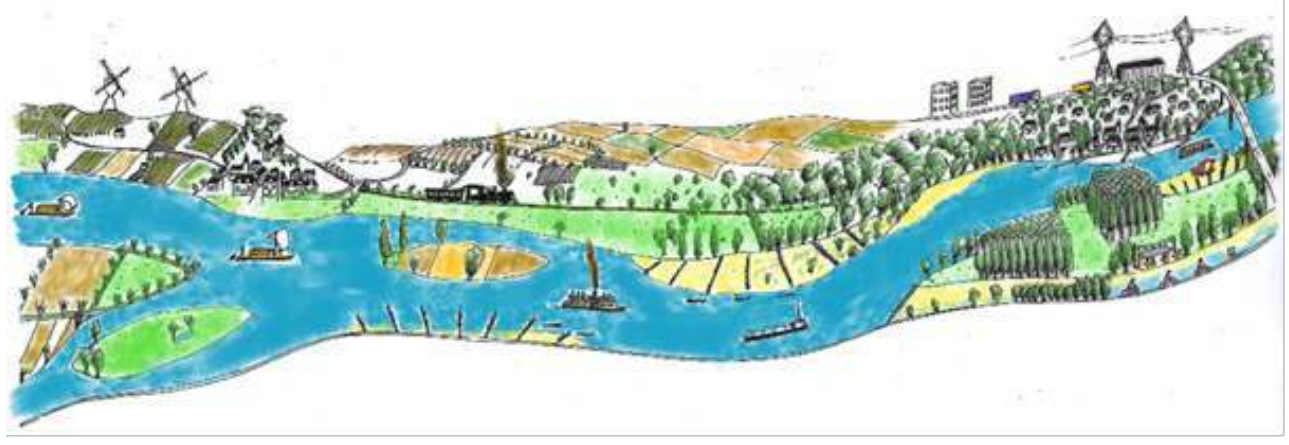

Source : Montembault, 2013, in Arnaud-Fessetta et al.

Bien qu'en contradiction avec les connaissances géohistoriques, cette représentation est le produit d'un processus d'artialisation et prend sens pour le grand public par comparaison avec d'autres fleuves, selon donc une naturalité relative (par exemple la Loire vis-à-vis du Rhône). Cette image est un support de marketing territorial efficace, exportant une marque-Loire qui associe le fleuve royal des châteaux avec le fleuve sauvage des grèves de sables. Si les touristes adhèrent à ce produit, la gestion publique doit en revanche réussir à placer le curseur entre préservation et évolution ${ }^{13}$ et articuler ces deux projets patrimoniaux. En 2000, l'inscription du Val de Loire au Patrimonial mondial permet cette articulation par la notion de paysage culturel (« CEuvres combinées de la nature et de l'homme») et opte pour une approche ouverte et dynamique du paysage (« Paysages culturels évolutifs et vivants »), non seulement adaptée à la longue histoire de la mise en valeur du fleuve et du Val, mais aussi à la dimension élargie du périmètre inscrit (le plus vaste en France, et qui intègre à l'aval une partie de Loire armoricaine, la section à épis entre Bouchemaine et Chalonnes).

Mais il n'en reste pas moins que ce libellé très ouvert doit se concrétiser par une gestion patrimoniale de la vallée. Or, tout processus de patrimonialisation implique une sélection (Di Méo, 1998), laquelle s'exerce ici dans le tracé même du périmètre Unesco ${ }^{14}$ et dans les dispositions prises par l'État ou les collectivités à travers le plan de gestion mis en œuvre aujourd'hui (et ses effets sur le droit des sols et la réglementation des usages ${ }^{15}$ ). Ce travail de sélection sur lequel s'appuie la valeur universelle exceptionnelle (VUE) justifiant l'inscription du Val de Loire et qui guide les réglementations (sur les objets et les usages à réguler ${ }^{16}$ ) et, là encore, en fonction d'un ou plusieurs état(s) de référence : la Renaissance pour le patrimoine bâti des châteaux (et jardins associés) et les paysages de la fin du XIX siècle pour l'écrin paysager dans lequel ils s'inscrivent. Ces paysages jardinés (la vallée de la Loire « jardin de la France) par une main-d'œuvre abondante (Montembault, 2002) sont 
la représentation qui sous-tend la patrimonialisation des paysages ligériens, en particulier celle des prairies alluviales.

Figure 4. Bloc diagramme de l'identité paysagère

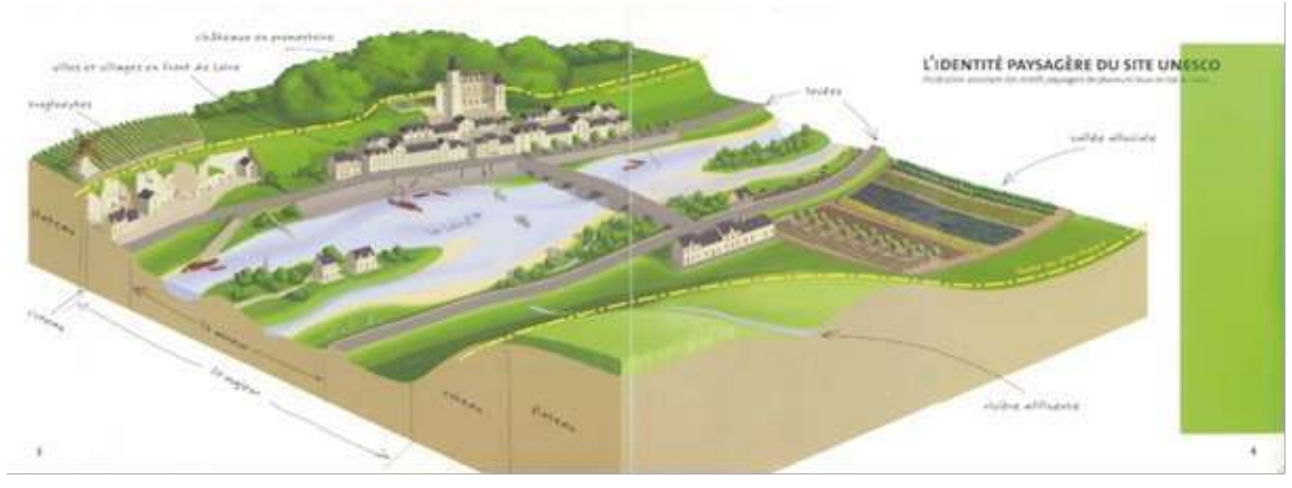

Source : Mission Loire, 2009, Vivre les paysages inscrits au patrimoine mondial de l'Unesco usages). Rarement explicite, mais toujours très opérant, il évoque un passé souvent idéalisé qui, justement parce qu'utopique, renvoie moins à un état antérieur qu'à un idéal futur. L'analyse du patrimoine articule donc nécessairement le présent, le passé et le futur à travers une chronogenèse qui donne du sens au temps présent en l'inscrivant dans une visée à la fois rétrospective, introspective et prospective (Héritier, 2013). Les épis de la Loire armoricaine entravent la linéarité de ces trois temporalités en apparaissant comme une parenthèse dans la longue histoire du fleuve. L'action publique consiste donc aujourd'hui à rétablir cette linéarité - gage d'un équilibre à retrouver à travers un rapport au temps stabilisé - en cherchant à les effacer matériellement. À défaut d'y parvenir, la représentation à l'œuvre du «fleuve sauvage » tend à les effacer immatériellement. L'action paysagère cherche donc à accommoder le paysage perçu et le paysage visible à travers une " reconquête » qui passe autant par les aménagements que par un récit qui, en dépit de la fiction qu'il construit, doit être pris au sérieux par le chercheur dans la mesure où il légitime l'aménagement. 


\section{BIBLIOGRAPHIE}

Baron-Yelles N., 2000, Recréer la nature. Écologie, paysage et société au marais d'Orx, Paris, ENS éditions, $216 \mathrm{p}$.

Barraud R., Carcaud N., Davodeau H., Montembault D., 2013-1, « Les épis de la Loire armoricaine, un héritage à la patrimonialité incertaine ", Norois n 228-2013/3: La patrimonialisation des paysages de l'eau dans l'Ouest de la France, p. 39-51.

Barraud R., Carcaud N., Davodeau H., Montembault D., Pordoy C., 2013-2, « Rivers groins along the Armoricain Loire : river responses and local resident reactions. A sociogeographical landscape reading as a complement for ecological engineering ", in Arnaud-Fassetta G., Masson E., Reynard E., European Continental Hydrosystems under Changing Water Policy, München, Verlag Dr.Friedrich Pfeil, p. 205-220.

Barraud R., Davodeau H., Montembault D., Taillade A., 2012, « Le remodelage des épis de la Loire armoricaine ; patrimoine naturel vs patrimoine culturel »? Les paysages de l'eau, édition électronique dirigée par G. Joly, Éditions du CTHS (actes du $135^{\mathrm{e}}$ congrès des sociétés historiques et scientifiques 6-11 avril 2010, Neuchâtel, Suisse), p. 7-20.

Barraud R., 2011, «Rivières du futur, wild rivers? », Vertigo - Revue électronique des sciences de l'environnement, hors série $\mathrm{n}^{\circ} 10$, [en ligne] : https://vertigo.revues.org/11411.

Barraud R., Germaine M.-A., 2017 (à paraître), Démanteler les barrages pour restaurer les cours d'eau. Controverse environnementale, représentations et projets, Versailles, Quæ.

Bercovitz R., 2015, Paysage, médiation paysagère et « bon état écologique » de la haute vallée de la Sèvre niortaise : mener une enquête historique pour fonder un projet partagé (XVIII ${ }^{e}$-XXI ${ }^{e}$ siècles), thèse de géographie, université Bordeaux III.

Blandin P., 2009, De la protection de la nature au pilotage de la biodiversité, Versailles, Quae, 124 p.

Bonin S., 2008, « La Loire-milieu, outil du développement durable », in Blanc N., Bonin S. (dir.), Grands barrages et habitants, Maison des sciences de l'homme, Versailles, Quæ, 336 p.

Bouleau G., Pont D., 2014, « Les conditions de référence de la directive-cadre européenne sur l'eau face à la dynamique des hydrosystèmes et des usages ", Natures, Sciences, Sociétés, vol. 22, n - 1, p. 3-14.

Bravard J.-P., 2003, « Dynamiques à long terme des systèmes écologiques ou de l'Eden impossible à la gestion de la variabilité », in Lévêque C., Van der Leeuw S. (éds.), Quelle nature voulons-nous?, Paris, Elsevier, p. 233-239.

Burnouf J., Carcaud N., Cubizolle H., Trément F., Visset L., Garcin M., Serieyssol K., 2001, « Les relations sociétés/milieux physiques depuis la fin du Tardiglaciaire : les apports du programme Loire ", Quaternaire, vol. 12, n 1, p. 5-13.

Burnouf J., Carcaud N., Garcin E., 2003, « La Loire entre mythes et réalités », 303, Arts, recherches et créations, La Loire, $\mathrm{n}^{\circ}$ 75, p. 13-17.

Carcaud N., Davodeau H., 2013, » Another Loire : The Armorican Loire ", in Landscapes and landforms of France, Springer Science+Business Media Dordrecht, p. 61-70. 
Charrier P., 1997, La vallée de la Loire des Ponts-de-Céà Bellevue (entre Anjou et Pays Nantais), évolutions et sensibilités, thèse de géographie, université de Nantes, $376 \mathrm{p}$.

Charrier P., 2000, «Entre Anjou et Pays nantais, interventions humaines et transformations hydro-morphologiques en Loire armoricaine (1750-1960) », Annales de géographie, 109, p. 115-131. Chouquer G., 2008, Traité d'archéogéographie - La crise des récits géohistoriques, Paris, Errance, 200 p. Chouquer G., Watteaux M., 2013, L'archéologie des disciplines géohistoriques. Traité d'archéogéographie - tome 2, Paris, Errance-Actes Sud., 408 p.

COLL (dir. Davodeau H.), 2012, Patrimoines et trajectoires paysagères des vallées ligériennes, rapport du programme de recherche PATRA 2010-2012, FEDER, Établissement public Loire, 286 p.

D’Andigné A., 1928, Essai sur la Loire navigable, Société d'éditions géographiques, maritimes et coloniales, Paris, $402 \mathrm{p}$.

Davodeau H., 2012, « Paysages et médiations, retour d'expériences pédagogiques », in Espace rural \& projet spatial vol. 3 - Du terrain à la recherche : objets et stratégies (dir. Guillot), p. 161-166/267.

Davodeau H., Pordoy C., Montembault D., Barraud R. Carcaud N., 2013, « Patrimonialiser la nature et les paysages : contradictions et paradoxes au sujet des épis de la Loire armoricaine ", L'Espace géographique, $\mathrm{n}^{\circ}$ 213/3, p. 241-253.

Delajartre A., 2013, « Le paysage est-il soluble dans le patrimoine écologique ? », Projets de paysage , $\mathrm{n}^{\circ} 15$.

Di Méo G., Géographie sociale et territoires, Paris, Nathan.

Droz Y., Miéville-Ott V. (dir.), 2005, La polyphonie du paysage, Lausanne, Presses polytechniques romandes, $227 \mathrm{p}$.

Dubois C., 2009, « Le paysage, enjeu et instrument de l'aménagement du territoire », Biotechnologie, Agronomie, Société, Environnement, décembre.

Ducamp R., 1900, «La navigabilité de Loire », Revue des eaux et forêts, t. 39, p. 586-591

Dufour S., Piégay H., 2009, « From the myth of a lost paradise to targeted river restoration : forget natural references and focus on human benefits ", River Research and Applications, vol. 25, Issue 5, p. 568-581.

Gallouedec L., 1897, « La Loire navigable », Annales de géographie, vol. 6, n 25, p. 45-60.

Haghe J.-P., 2010, Penser l'eau : contribution à une généalogie des idées à travers l'exemple français, in Schneier-Madanes G. (dir.), L'eau mondialisée. La gouvernance en question, Paris, La Découverte, p. 47-60.

Héritier S., 2013, « Le patrimoine comme chronogenèse. Réflexions sur l'espace et le temps », Annales de géographie, $\mathrm{n}^{\circ}$ 689-1, p. 3-23.

Jacob N., 2009, « Géohistoire/géo-histoire : quelles méthodes pour quel récit », Géocarrefour, vol. $84, \mathrm{n}^{\circ} 4$, p. 211-216.

Larrère R. et C., 1997, Du bon usage de la nature - Pour une philosophie de l'environnement, Paris, Aubier, coll. « Alto».

Le Marec Y., 2000, « Construire leur accord. Ingénieurs des Ponts et Chaussées et paysans dans l'estuaire de la Loire (xIX ${ }^{\mathrm{e}}-\mathrm{XX}^{\mathrm{e}}$ siècles) », Genèses, vol. 40, n 1, p. 108-130. 
Lecœur Y., 2011, » La Loire moyenne naviguée au XIX ${ }^{\mathrm{e}}$ siècle : représentations, réglementation et aménagement », Vertigo - La revue électronique en sciences de l'environnement [en ligne] : http:// vertigo.revues.org/11371; DOI : 10.4 000/vertigo.11371

Lévêque C., Van der Leeuw S. (éds.), 2006, Temps et espaces des crises de l'environnement, Versailles, Quæ, 408 p.

Mantellier P., 1864, Histoire de la communauté des marchands fréquentant la rivière de Loire et fleuves descendant en icelle, tome 1, Paris, Imp. G. Jacob., 501 p.

Moatar F., Dupont N. (coord.), 2016, La Loire fluviale et estuarienne - Un milieu en évolution, Versailles, Quæ, 320 p.

Montembault D., 2002, Les vallées face à l'appropriation urbaine, thèse de géographie non publiée, dirigée par le prof. J.-B., Humeau, université d'Angers, 2002.

Montembault D., Toublanc M., Davodeau H., Geisler E., Leconte L., Romain F., Luginbuhl A., Guttinger P., 2015, Participation et renouvellement des pratiques paysagistes, in Biodiversité, paysage et cadre de vie. La démocratie en pratique, , Paris, Victoires Édition, p. 171-187/287.

Pelosse V., Micoud A. 1993, « Introduction : du domestique au sauvage cultivé : des catégories pertinentes de la biodiversité ? ", Études rurales, vol. 129, n 1, p. 9-14.

Poligot-Pitsch S., Geffray G., Pichon N., 2014, « Contribution de l'hydrométrie au suivi de l'évolution du lit de la Loire aval », La Houille blanche, 5, p. 24-30

Rode S., 2010, « De l'aménagement au ménagement des cours d'eau : le bassin de la Loire, miroir de l'évolution des rapports entre aménagement fluvial et environnement », Cybergeo - revue européenne de géographie, article 506 [en ligne] : https://cybergeo.revues.org/23253.

Schwob M., 1900, La Loire navigable, Communication faite au congrès national des travaux publics, Nantes, M. Schwob et fils, $80 \mathrm{p}$.

Sgard A., 2010, «Le paysage dans l'action publique : du patrimoine au bien commun », Développement durable et Territoires, vol. $1, \mathrm{n}^{\circ} 2$.

Vauthier-Vézier A., 2007, L'estuaire et le port, L'identité maritime de Nantes au XIXe siècle, PUR, Rennes, 236 p.

Zimmermann M., 1905, « La « Loire navigable », le « projet de Grand Central » et l'avenir du port de Nantes », Annales de géographie, t. 14, n 73, p. 84-87.

\section{NOTES}

1. Patrimonialisation des paysages de Loire (PATRA, 2010/2012, dir. H. Davodeau), Les paysages de vallée face au processus de restauration écologique - Le cas des démantèlements de barrages (REPPAVAL, 2012/2015, dir. R. Barraud et M.-A. Germaine), Expérimenter la participation dans le projet de paysage (PDD2, 2011/2014, dir. D. Montembault).

2. La secteur d'expérimentation permet d'observer l'amorce d'un rééquilibrage avec un relèvement du niveau moyen à l'étiage de l'ordre de $30 \mathrm{~cm}$ (Poligot-Pitsch et al., 2014).

3. Le bateau à aubes Loire Princesse est maintenant bien connu des riverains, tant par son gabarit imposant que par les problèmes de navigation dont la presse locale se délecte : « Loire-Princesse : le fleuve sauvage plus fort que le bateau de croisière » (France 3, 8 juin 2015).

4. http://www.uneautreloire.fr/ 
5. En Maine-et-Loire, où les communes nouvelles sont nombreuses, la place du paysage dans les nouvelles appellations est significative: http://www.maine-et-loire.gouv.fr/la-reformeterritoriale-en-maine-et-loire-a3803.html

6. « [...] quand je me promène sur les bords de la Loire, je m'étonne de l'absence de jeux et de cris d'enfants là où, à 8 , à 10 ans, nos bandes menaient leur train sur le quai et le long des buissons de la rive. À cette époque, on construisait les épis noyés de la Loire navigable: de hautes piles de claies de châtaignier s'entassaient sur la cale: le grand jeu était d'y grimper et de sauter sur le pavé du quai de la plus grande hauteur possible » (Gracq, Lettrines 2, 1974).

7. Objectifs du programme (62,6 millions d'euros): rééquilibrage longitudinal du fleuve (réduction de la pente de la ligne d'eau et du fond), le rééquilibrage latéral du lit mineur (extension de l'espace de mobilité), amélioration de la connectivité des annexes hydrauliques et des bras secondaires en fonction des débits de la Loire, amélioration du fonctionnement actuel des annexes par des travaux de restauration, suivis d'éventuels travaux d'entretien.

8. ACT Consultants, "Étude des possibilités de développement du fret fluvial sur la Loire», Rapport final, juillet 2009, 199 p.

9. Le point de vue du CLD sur les actions programmées dans le cadre du Plan Loire 4 est globalement positif, mais l'association conserve un positionnement critique, bien que constructif : $\quad$ http://www.loire-de-demain.fr/wp-content/uploads/2015/01/2013-02-CLDRegard_sur_PlanLoire4.pdf.

10. Le comité Loire de demain a été créé en 2005 et regroupe 350 particuliers, 33 associations et 24 communes riveraines : http://www.loire-de-demain.fr/

11. "Instrument de médiation pour communiquer le territoire, pour interpeller la population sur la question de son cadre de vie et lui laisser en débattre intuitivement, ainsi que pour engager un dialogue citoyen entre décideurs, acteurs et populations quant aux enjeux et actions de développement territorial, le paysage peut donc servir un aménagement plus démocratique du territoire [...] Ainsi convié, le paysage constitue des discussions plus larges que strictement paysagères, relatives au territoire et à l'environnement. "

12. «[...] transmettre un paysage bien commun signifie selon nous transmettre le lien, la force du lien » (Sgard, 2010).

13. "L'authenticité de ce paysage tient dans sa capacité à se renouveler et à s'adapter tout en ayant su préserver, au fil des siècles, les patrimoines civils et religieux ainsi que les grands équilibres environnementaux qui font l'exceptionnelle qualité de ce site ", Les cahiers du Val de Loire patrimoine mondial, $\mathrm{n}^{\circ} 4$, p. 9.

14. Duquel est exclue la centrale électronucléaire de Saint-Laurent-des-Eaux qui était pourtant intégrée au premier périmètre en tant qu'élément de modernité exprimant, à sa manière, le lien tissé entre le fleuve et les hommes.

15. L'inscription au Patrimoine mondial n'induit pas de réglementation contraignante : ce n'est qu'indirectement qu'elle devient opposable, lorsque ses orientations sont relayées dans les documents d'urbanisme réglementaires ou lorsque les services de l'État s'appuient sur ces contenus pour argumenter une position (par ex.: contre l'autorisation de creusement d'une baignade sur la grève).

16. «Cinq facteurs de risques à maîtriser : les mutations agricoles, l'étalement urbain, les zones d'activités mal intégrées, les grandes infrastructures ou grands équipements hors contexte, l'architecture banale » (L'Attitude ligérienne, Faire projet(s) en Val de Loire, référentiel urbanisme-aménagement, Agences d'urbanisme d'Angers, Orléans et Tous, avec la Mission Val de Loire, 2012, 35 p.

17. Voir l'exposition permanente de la maison du Parc naturel régional Loire Anjou Touraine à Montsoreau (49).

18. «[...] le paysage naturalisé n'est autre que l'illusion d'un paysage donné objectivement, d'un paysage évident, qui va de soi [...] (Droz et al., 2009, p. 14). 


\section{RÉSUMÉS}

Cet article revient sur les résultats d'un programme de recherche portant sur la patrimonialisation des paysages de Loire. Nous questionnons la position du chercheur face à un patrimoine encore "dormant», mais dont il anticipe l'émergence prochaine. Une contextualisation des épis de navigation dans l'histoire récente du fleuve permet de comprendre la constitution de ces héritages. Puis nous présentons les résultats d'un travail d'enquête auprès des riverains pour démontrer leur faible connaissance de cette histoire en pointant les paradoxes de leurs représentations sur ces objets pour, in fine, rassembler les indices permettant de formuler l'hypothèse de l'émergence prochaine de leur valeur patrimoniale. Dans la perspective d'une controverse à venir, nous défendons une posture de recherche «par le paysage » sur le patrimoine et la participation, susceptible de mettre en débat les temporalités à l'œuvre dans la politique de restauration du fleuve Loire.

This paper is based on the findings of a research project dealing landscape heritage of the Loire valley (France). We question the position of the scientist facing what we're calling here a 'latent' heritage. The first part of the paper provides a geo-historical contextualisation of the hydraulic works (groynes) intended to improve navigation (channeling). We focus on identifying and analyzing time references used over the different periods : before, during and after the initial development of the groynes and the channel. Then, we present the results of a survey work with local residents demonstrating their weak knowledge of this history by pointing out the paradoxes of their social representations of these objects. The perspective of a future controversy, we defend a position of research "based on landscape analysis" to deal with heritage and participation issues in order to debate the temporalities at work in the restoration policy of the Loire River.

\section{INDEX}

Keywords : landscape, heritage, temporalities, mediation, forward-looking analysis, Loire River Mots-clés : paysage, patrimoine, temporalités, médiation, prospective, Loire

\section{AUTEURS}

\section{HERVÉ DAVODEAU}

Hervé Davodeau est maître de conférences en géographie à Agrocampus Ouest Angers où il enseigne auprès des ingénieurs paysagistes. Ses travaux conduits au sein de l'UMR ESO portent sur l'action paysagère (politiques publiques, pratiques professionnelles, mobilisations sociales). Il a coordonné le programme PATRA, participe au programme Didactique du paysage, et est membre du comité de rédaction de la revue Projets de paysage. herve.davodeau@agrocampusouest.fr 


\section{RÉGIS BARRAUD}

Régis Barraud est maître de conférences en géographie à l'université de Poitiers. Ses recherches sont effectuées au sein du laboratoire RURALITES (EA 2252) et portent sur : 1- Les dynamiques paysagères (structures, usages et représentations sociales) ; 2- La construction sociale de la nature sauvage. Il a coordonné, avec Marie-Anne Germaine (université de Paris Ouest Nanterre, LAVUE UMR CNRS), le programme REPPAVAL. regis.barraud@univ-poitiers.fr http://reppaval.hypotheses.org 